\title{
Gamma-Ray Bursts and Bursters
}

\author{
P. Mészáros ${ }^{a * \dagger}$

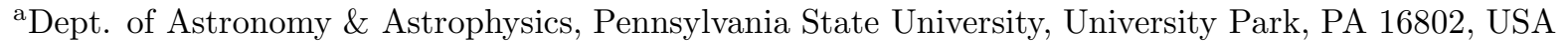

\begin{abstract}
Major advances have been made in the field of gamma-ray bursts in the last two years. The successful discovery of X-ray, optical and radio afterglows, which were predicted by theory, has made possible the identification of host galaxies at cosmological distances. The energy release inferred in these outbursts place them among the most energetic and violent events in the Universe. Current models envisage this to be the outcome of a cataclysmic event leading to a relativistically expanding fireball, in which particles are accelerated at shocks and produce nonthermal radiation. The substantial agreement between observations and the theoretical predictions of the standard fireball shock model provide confirmation of the basic aspects of this scenario. The continued observations show a diversity of behavior, providing valuable constraints for more detailed, post-standard models which incorporate more realistic physical features. Crucial questions being now addressed are the beaming at different energies and its implications for the energetics, the time structure of the afterglow, its dependence on the central engine or progenitor system behavior, and the role of the environment on the evolution of the afterglow.
\end{abstract}

\section{INTRODUCTION}

The discovery of gamma-ray bursts (GRB) by the Vela military test-ban treaty satellites was announced in 1973 45, and was quickly confirmed by Soviet Konus satellite measurements 52. Then, for 23 years GRB remained essentially just that: brief outbursts of gamma-rays which pierced, for a brief instant, an otherwise pitch-black gamma-ray sky. An intense debate festered for a long time on whether they were objects in our galaxy or at cosmological distances. The first major breakthrough came in 1992 with the launch of the Compton Gamma-Ray Observatory, whose superb results are summarized in a review by Fishman \& Meegan [23]. In particular the all-sky survey from the BATSE instrument showed that bursts were isotropically distributed, strongly suggesting either a cosmological or an extended galactic halo distribution, with essentially zero dipole and quadrupole components. The spectra are definitely non-thermal, typically fitted in the $\mathrm{MeV}$ range by broken power-laws

\footnotetext{
*Also Institute for Theoretical Physics, UCSB, and Ctr. for Gravitational Physics \& Geometry, Penn State Univ. Supported by NASA NAG-5 2857 and NSF PHY94-07194 ${ }^{\dagger}$ Plenary talk given at the 19th Texas Symp. on Relativistic Astrophysics \& Cosmology, Paris, Dec. 1998
}

whose energy per decade $\nu F_{\nu}$ peak is in the range 50-500 KeV [3], the power law sometimes extending to $\mathrm{GeV}$ energies [38]. GRB appeared to leave no detectable traces at other wavelengths, except in some cases briefly in X-rays [119,13]. The gamma-ray durations range from $10^{-3} \mathrm{~s}$ to about $10^{3} \mathrm{~s}$, with a roughly bimodal distribution of long bursts of $t_{b} \gtrsim 2 \mathrm{~s}$ and short bursts of $t_{b} \lesssim 2 \mathrm{~s}$ [48], and substructure sometimes down to milliseconds. The gamma-ray light curves range from smooth, fast-rise and quasi-exponential decay (FREDs), through curves with several peaks, to highly variable curves with many peaks [23, 49]. The pulse distribution is complex 90,72, and the time histories can provide clues for the geometry of the emitting regions [21,22].

Theoretically, it was clear from early on that if GRB are cosmological, enormous energies are liberated in a small volume in a very short time, and an $e^{ \pm}-\gamma$ fireball must form [78,32, 117], which would expand relativistically. The main difficulty with this was that a smoothly expanding fireball would convert most of its energy into kinetic energy of accelerated baryons (rather than photons), and would produce a quasi-thermal spectrum, while the typical timescales would not explain events much longer than milliseconds. This 
problem was solved with the introduction of the "fireball shock model" 98,57, based on the realization that shocks are likely to arise, at the latest when the fireball runs into an external medium, which would occur after the fireball is optically thin and would reconvert the kinetic energy into nonthermal radiation. The complicated light curves can be understood in terms of internal shocks [99] in the outflow itself, before it runs into the external medium, caused by velocity variations in the outflow from the source,

The next major breakthrough came in early 1997 when the Italian-Dutch satellite Beppo-SAX succeeded in providing accurate X-ray measurements which, after a delay of 4-6 hours for processing, led to positions [14], allowing follow-ups at optical and other wavelengths, e.g. [127]. This paved the way for the measurement of redshift distances, the identification of candidate host galaxies, and the confirmation that they were indeed at cosmological distances 66, 18, 50. The detection of other GRB afterglows followed in rapid succession, sometimes extending to radio 24, 25] and over timescales of many months [128, and in a number of cases resulted in the identification of candidate host galaxies, e.g. [108,9,74, etc. The study of afterglows has provided strong confirmation for the generic fireball shock model of GRB. This model in fact led to a correct prediction 61, in advance of the observations, of the quantitative nature of afterglows at wavelengths longer than $\gamma$-rays, which were in substantial agreement with the data 129,121, 132, 101, 136.

A major issue raised by the measurement of large redshifts, e.g. 50,51, is that the measured $\gamma$-ray fluences imply a total energy of order $10^{54}\left(\Omega_{\gamma} / 4 \pi\right)$ ergs, where $\Delta \Omega_{\gamma}$ is the solid angle into which the gamma-rays are beamed. A beamed jet would clearly alleviate the energy requirements, but it is only recently that tentative evidence has been reported for evidence of a jet [51,27,12]. Whether a jet is present or not, such energies are possible [62] in the context of compact mergers involving neutron star-neutron star (NS-NS ) or black hole-neutron star (BH-NS ) binaries, or in hypernova/collapsar models involving a massive stellar progenitor 81,95]. In both cases, one is led to rely on MHD extraction of the spin energy of a disrupted torus and/or a central fast spinning $\mathrm{BH}$, which can power a relativistic fireball resulting in the observed radiation.

While it is at present unclear which, if any, of these progenitors is responsible for GRB, or whether perhaps different progenitors represent different subclasses of GRB, there is general agreement that they all would be expected to lead to the generic fireball shock scenario mentioned above. Much of the current effort is dedicated to understanding the progenitors more specifically, and trying to determine what effect, if any, they have on the observable burst and afterglow.

\section{Black Hole/Debris systems as generic GRB energy sources}

It has become increasingly apparent in the last few years that most plausible GRB progenitors suggested so far are expected to lead to a system with a central $\mathrm{BH}$ plus a temporary debris torus around it. Scenarios leading to this include, e.g. NS-NS or NS-BH mergers, Helium core - black hole $[\mathrm{He} / \mathrm{BH}]$ or white dwarf - black hole [WD$\mathrm{BH}]$ mergers, and the wide category labeled as hypernova or collapsars including failed supernova Ib [SNe Ib], single or binary Wolf-Rayet [WR] collapse, etc. [80, 137, 62, 81, 95, and accretioninduced collapse [130,96]. An important point is that the overall energetics from these various progenitors do not differ by more than about one order of magnitude 66]. Another possibility is massive black holes $\left(\sim 10^{3}-10^{5} M_{\odot}\right)$ in the halos of galaxies. Some related models involve a compact binary or a temporarily rotationally stabilized neutron star, perhaps with a superstrong field, e.g. [125, 123.130,118], which ultimately also should lead to a $\mathrm{BH}$ plus debris torus.

Two large reservoirs of energy are available in these systems: the binding energy of the orbiting debris, and the spin energy of the black hole [62]. The first can provide up to $42 \%$ of the rest mass energy of the disk, for a maximally rotating black hole, while the second can provide up to $29 \%$ of the rest mass of the black hole itself. The question is how to extract this energy.

One energy extraction mechanisms is the $\nu \bar{\nu} \rightarrow$ $e^{+} e^{-}$process [20], which can tap the thermal 
energy of the torus produced by viscous dissipation. To be efficient, the neutrinos must escape before being advected into the hole; on the other hand, the efficiency of conversion into pairs (which scales with the square of the neutrino density) is low if the neutrino production is too gradual. Typical estimates suggest a fireball of $\lesssim 10^{51}$ erg [106,28, 53, except perhaps in the collapsar case where [95] estimate $10^{52.3}$ ergs for optimum parameters. If the fireball is collimated into a solid angle $\Omega_{j}$ then of course the apparent "isotropized" energy would be larger by a factor $\left(4 \pi / \Omega_{j}\right)$, but unless $\Omega_{j}$ is $\lesssim 10^{-3}-10^{-4}$ this would fail to satisfy the apparent isotropized energy of $4 \times 10^{54}$ ergs deduced for GRB 990123 51 .

An alternative, and more efficient mechanism for tapping the energy of the torus may be through dissipation of magnetic fields generated by the differential rotation in the torus 80, 71,62 , 43]. Even before the BH forms, a NS-NS merging system might lead to winding up of the fields and dissipation in the last stages before the merger 55, 129].

However, a larger energy source is available in the hole itself, especially if formed from a coalescing compact binary, since then it is guaranteed to be rapidly spinning. Being more massive, it could contain more energy than the torus. The energy extractable in principle through MHD coupling to the rotation of the hole by the B-Z (Blandford \& Znajek (5)) effect could then be even larger than that contained in the orbiting debris 62 81]. Collectively, any such MHD outflows have been referred to as Poynting jets.

The various progenitors differ only slightly in the mass of the $\mathrm{BH}$ and that of the debris torus they produce, but they may differ more markedly in the amount of rotational energy contained in the BH. Strong magnetic fields, of order $10^{15}$ $\mathrm{G}$, are needed needed to carry away the rotational or gravitational energy in a time scale of tens of seconds 125, 123, which may be generated on such timescales by a convective dynamo mechanism, the conditions for which are satisfied in freshly collapsed neutron stars or neutron star tori 19,46]. If the magnetic fields do not thread the BH, then a Poynting outflow can at most carry the gravitational binding energy of the torus. For a maximally rotating and for a slow-rotating $\mathrm{BH}$ this is

$E_{t}=\epsilon M_{\odot} c^{2} \begin{cases}0.42\left(M_{d} / M_{\odot}\right) \operatorname{ergs}, & \text { (fast rot.); } \\ 0.06\left(M_{d} / M_{\odot}\right) \text { ergs }, & \text { (slow rot.), }\end{cases}$

where $\epsilon$ is the efficiency in converting gravitational into MHD jet energy. The torus or disk mass in a NS-NS merger is 107 $M_{d} \sim 10^{-1}$ $10^{-2} M_{\odot}$, and for a NS-BH, a He-BH, WD-BH merger or a binary WR collapse it may be estimated at 81,28] $M_{d} \sim 1 M_{\odot}$. In the HeWD-BH merger and WR collapse the mass of the disk is uncertain due to lack of calculations on continued accretion from the envelope, so $1 M_{\odot}$ is just a rough estimate. The maximum torus-based MHD energy extraction is then

$E_{\text {max }, t} \sim\left\{\begin{array}{l}8 \times 10^{53} \epsilon\left(M_{d} / M_{\odot}\right) \text { ergs }, \\ 1.2 \times 10^{53} \epsilon\left(M_{d} / M_{\odot}\right) \text { ergs }, \\ 0.8 \times 10^{53} \epsilon\left(M_{d} / 0.1 M_{\odot}\right) \text { ergs }, \quad ;\end{array} ;\right.$

for the NS-BH, He/WD-BH or collapsar case; the (slow rotating) failed SN Ib case; and NS-NS case, respectively.

If the magnetic fields in the torus thread the $\mathrm{BH}$, the spin energy of the $\mathrm{BH}$ can in principle be extracted via the 榪 (B-Z) mechanism ([62]). The extractable energy is

$E_{b h} \sim \epsilon f(a) M_{b h} c^{2}$,

where $\epsilon$ is the MHD efficiency factor, $f(a)=$ $1-\left(\left[1+\sqrt{1-a^{2}}\right] / 2\right)^{1 / 2} \leq 0.29$ is the rotational efficiency factor, and $a=J c / G M^{2}$ is the rotation parameter, which equals 1 for a maximally rotating black hole. The $f(a)$ rotational factor is is small unless $a$ is close to 1 , where it rises sharply to its maximum value $f(1)=0.29$, so the main requirement is a rapidly rotating black hole, $a \gtrsim 0.5$. For a maximally rotating $\mathrm{BH}$, the extractable energy is therefore

$E_{\text {max }, b h} \sim 0.29 \epsilon M_{b h} c^{2} \sim 5 \times 10^{53} \epsilon\left(M_{b h} / M_{\odot}\right)$ ergs.(3)

Rapid rotation is guaranteed in a NS-NS merger, since the radius (especially for a soft equation of state) is close to that of a black hole and the final orbital spin period is close to the required maximal spin rotation period. The central 
BH will have a mass 106, 107 of about $2.5 M_{\odot}$, so the NS-NS system can power a jet of up to $E_{N S-N S} \lesssim 1.3 \times 10^{54} \epsilon\left(M_{b h} / 2.5 M_{\odot}\right)$ ergs. A maximal rotation rate may also be possible in a He$\mathrm{BH}$ merger, depending on what fraction of the He core gets accreted along the rotation axis as opposed to along the equator [28], and the same should apply to the binary fast-rotating WR scenario, which probably does not differ much in its final details from the He-BH merger. For a fast rotating $\mathrm{BH}$ of $2.5-3 M_{\odot}$ threaded by the magnetic field, the maximal energy carried out by the jet is then similar or somewhat larger than in the NS-NS case. The scenarios less likely to produce a fast rotating $\mathrm{BH}$ are the NS-BH merger (where the rotation parameter could be limited to $a \leq M_{n s} / M_{b h}$, unless the $\mathrm{BH}$ is already fastrotating) and the failed $\mathrm{SNe} \mathrm{Ib}$ (where the last material to fall in would have maximum angular momentum, but the material that was initially close to the hole has less angular momentum). The electromagnetic energy extraction from the $\mathrm{BH}$ in these could be limited by the $f(a)$ factor, but a lower limit would be given by the energy available from the gravitational energy of the disk, in the second line of equation (11).

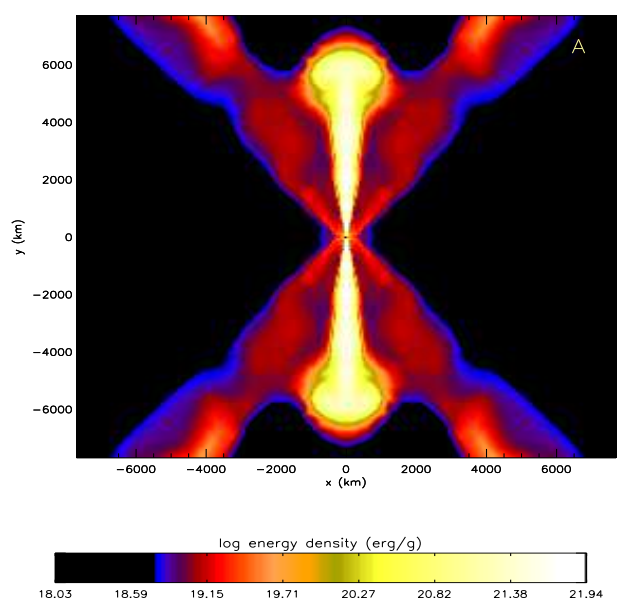

Figure 1. Jet formation in a collapsar model leading to black hole from collapse of a fast rotating He core [53]
The total energetics differ thus between the various models at most by a factor 20 for a Poynting (MHD) jet powered by the torus binding energy, whereas for Poynting jets powered by the $\mathrm{BH}$ spin energy they differ at most by a factor of a few, depending on the rotation parameter. For instance, allowing for a total efficiency of $50 \%$, a NS-NS merger whose jet is powered by the torus binding energy would require a beaming of the $\gamma$ rays by a factor $\left(4 \pi / \Omega_{j}\right) \sim 100$, or beaming by a factor $\sim 10$ if the jet is powered by the B-Z mechanism, to produce the equivalent of an isotropic energy of $4 \times 10^{54}$ ergs. The beaming requirements of BH-NS and some of the collapsar scenarios are even less constraining, either when tapping the torus or the BH. Thus, even the most extreme energy requirements inferred observationally thus far can be plausibly satisfied by scenarios leading to a $\mathrm{BH}$ plus torus system.

The major difference between the various models is expected to be in the location where the burst occurs relative to the host galaxy (see $\delta$ []). They are also likely to differ substantially in the efficiency of producing a directly observable relativistic outflow, as well as in the amount of collimation of the jet they produce. The conditions for the efficient escape of a high- $\Gamma$ jet are less propitious if the "engine" is surrounded by an extensive envelope. In this case the jet has to "punch through" the envelope, and its ability to do so may be crucially dependent on the level of viscosity achieved in the debris torus (e.g. [53]), higher viscosities leading to more powerful jets (see Figure (1). The simulations, so far, are nonrelativistic and one can only infer that high enough viscosities can lead to jets capable of punching though a massive (several $M_{\odot}$ ) envelope. This is facilitated, of course, if the envelope is fast-rotating, as in this case there is a centrifugally induced column density minimum along the spin axis, which might be small enough to allow punch-through to occur. If they do, a very tightly collimated beam may arise. "Cleaner" environments, such as NS-BH or NS-NS merger, or rotational support loss/accretion induced collapse to $\mathrm{BH}$ would have much less material to be pushed out of the way by a jet, while their energy is, to order of magnitude, similar to that in massive stellar pro- 
genitor cases. In these cases, on the other hand, there is no natural choke to collimate a jet, which might therefore be somewhat wider than in massive progenitor cases.

\section{The Fireball Shock Scenario}

Irrespective of the details of the progenitor, the resulting fireball is expected to be initially highly optically thick. From causality considerations the initial dimensions must be of order $c t_{v a r} \lesssim 10^{7} \mathrm{~cm}$, where $t_{v a r}$ is the variability timescale, and the luminosities must be much higher than a solar Eddington limit. Since most of the spectral energy is observed above $0.5 \mathrm{MeV}$, the optical depth against $\gamma \gamma \rightarrow e^{ \pm}$is large, and an $e^{ \pm}, \gamma$ fireball is expected. Due to the highly super-Eddington luminosity, this fireball must expand. Since in many bursts one observes a large fraction of the total energy at photon energies $\epsilon_{\gamma} \gtrsim 1 \mathrm{GeV}$, somehow the flow must be able to avoid degrading these photons $\left(\gamma \gamma \rightarrow e^{ \pm}\right.$would lead, in a stationary or slowly expanding flow, to photons just below $0.511 \mathrm{MeV}[34]$ ). In order to avoid this, it seems inescapable that the flow must be expanding with a very high Lorentz factor, since in this case the relative angle at which the photons collide is less than $\Gamma^{-1}$ and the threshold for the pair production is effectively diminished. The bulk Lorentz factor must be

$\Gamma \gtrsim 10^{2}\left(\epsilon_{\gamma, 10 \mathrm{GeV}} \epsilon_{t, \mathrm{MeV}}\right)^{1 / 2}$,

in order for photons with energy $\epsilon_{\gamma} \gtrsim 10 \mathrm{GeV}$ to escape annihilation against target photons of energy $\epsilon_{t} \sim 1 \mathrm{MeV}$ [54]34. Thus, simply from observations and general physical considerations, a relativistically expanding fireball is expected. ¿From general considerations [58], one can see that an outflow arising from an initial energy $E_{o}$ imparted to a mass $M_{o}<<E_{0} / c^{2}$ within a radius $r_{l}$ will lead to an expansion. Initially the bulk Lorentz $\Gamma \propto r$, while comoving temperature drops $\propto r^{-1}$; however, $\Gamma$ cannot increase beyond $\Gamma_{\max } \sim \eta \sim E_{o} / M_{o} c^{2}$, which is achieved at a radius $r / r_{l} \sim \eta$, beyond which the flow continues to coast with $\Gamma \sim \eta \sim$ constant [58].

$\Gamma \sim\left\{\begin{array}{ll}\left(r / r_{l}\right), & \text { for } r / r_{l} \lesssim \eta \\ \eta, & \text { for } r / r_{l} \gtrsim \eta\end{array}\right.$.
However, the observed $\gamma$-ray spectrum observed is generally a broken power law, i.e., highly nonthermal. The optically thick $e^{ \pm} \gamma$ fireball cannot, by itself, produce such a spectrum (it would tend rather to produce a modified blackbody, [78, 32]). In addition, the expansion would lead to a conversion of internal energy into kinetic energy of expansion, so even after the fireball becomes optically thin, it would be highly inefficient, most of the energy being in the kinetic energy of the associated protons, rather than in photons.

The most likely way to achieve a nonthermal spectrum in an energetically efficient manner is if the kinetic energy of the flow is re-converted into random energy via shocks, after the flow has become optically thin [98]. This is a plausible scenario, in which two cases can be distinguished. In the first case (a) the expanding fireball runs into an external medium (the ISM, or a pre-ejected stellar wind [98,57, 41, 109]. The second possibility (b) is that 99,77, even before external shocks occur, internal shocks develop in the relativistic wind itself, faster portions of the flow catching up with the slower portions. This is a completely generic model, which is independent of the specific nature of the progenitor, as long as it delivers the appropriate amount of energy ( $\left.\gtrsim 10^{52} \mathrm{erg}\right)$ in a small enough region $\left(\lesssim 10^{7} \mathrm{~cm}\right)$. This model has been successful in explaining the major observational properties of the gamma-ray emission, and is the main paradigm used for interpreting the GRB observations.

External shocks will occur in an impulsive outflow of total energy $E_{o}$ in an external medium of average particle density $n_{o}$ at a radius and on a timescale

$r_{d e c} \sim \quad 10^{17} E_{53}^{1 / 3} n_{o}^{-1 / 3} \eta_{2}^{-2 / 3} \mathrm{~cm}$,

$t_{\text {dec }} \sim r_{\text {dec }} /\left(c \Gamma^{2}\right) \sim 3 \times 10^{2} E_{53}^{1 / 3} n_{o}^{-1 / 3} \eta_{2}^{-8 / 3} \mathrm{~s}$,

where the lab-frame energy of the swept-up external matter $\left(\Gamma^{2} m_{p} c^{2}\right.$ per proton) equals the initial energy $E_{o}$ of the fireball, and $\eta=\Gamma=10^{2} \eta_{2}$ is the final bulk Lorentz factor of the ejecta. The typical observer-frame dynamic time of the shock (assuming the cooling time is shorter than this) is $t_{d e c} \sim r_{d e c} / c \Gamma^{2} \sim$ seconds, for typical parameters, and $t_{b} \sim t_{d e c}$ would be the burst duration 
(the impulsive assumption requires that the initial energy input occur in a time shorter than $\left.t_{d y n}\right)$. Variability on timescales shorter than $t_{d e c}$ may occur on the cooling timescale or on the dynamic timescale for inhomogeneities in the external medium, but generally this is not ideal for reproducing highly variable profiles 111. However, it can reproduce bursts with several peaks 83 and may therefore be applicable to the class of long, smooth bursts.

The same behavior $\Gamma \propto r$ with comoving temperature $\propto r^{-1}$, followed by saturation $\Gamma_{\max } \sim \eta$ at the same radius $r / r_{l} \sim \eta$ occurs in a wind scenario [79], if one assumes that a lab-frame luminosity $L_{o}$ and mass outflow $\dot{M}_{o}$ are injected at $r \sim r_{l}$ and continuously maintained over a time $t_{w}$; here $\eta=L_{o} / \dot{M}_{o} c^{2}$. In such wind model, internal shocks will occur at a radius and over a timescale [99]

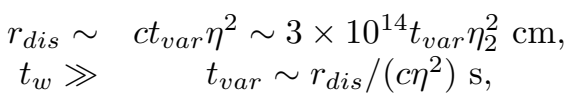

where shells of different energies $\Delta \eta \sim \eta$ initially separated by $c t_{v}$ (where $t_{v} \leq t_{w}$ is the timescale of typical variations in the energy at $r_{l}$ ) catch up with each other. In order for internal shocks to occur above the wind photosphere $r_{p h} \sim$ $\dot{M} \sigma_{T} /\left(4 \pi m_{p} c \Gamma^{2}\right)=1.2 \times 10^{14} L_{53} \eta_{2}^{-3} \mathrm{~cm}$, but also at radii greater than the saturation radius (so that most of the energy does not come out in the photospheric quasi-thermal radiation component) one needs to have $7.5 \times 10^{1} L_{51}^{1 / 5} t_{\text {var }}^{-1 / 5} \lesssim \eta 3 \times$ $10^{2} L_{53}^{1 / 4} t_{\text {var }}^{-1 / 4}$. This type of models have the advantage 99 that they allow an arbitrarily complicated light curve, the shortest variation timescale $t_{\text {var }} \gtrsim 10^{-3} \mathrm{~s}$ being limited only by the dynamic timescale at $r_{l}$, where the energy input may be expected to vary chaotically. Such internal shocks have been shown explicitly to reproduce (and be required by) some of the more complicated light curves 111, 47, 87] (see however [16]).

\section{The Simple Standard Afterglow Model}

The dynamics of GRB and their afterglows can be understood in a fairly simple manner, independently of any uncertainties about the progen- itor systems, using a generalization of the method used to model supernova remnants. The simplest hypothesis is that the afterglow is due to a relativistic expanding blast wave, which decelerates as time goes on 61. The complex time structure of some bursts suggests that the central trigger may continue for up to 100 seconds, the $\gamma$-rays possibly being due to internal shocks. However, at much later times all memory of the initial time structure would be lost: essentially all that matters is how much energy and momentum has been injected; the injection can be regarded as instantaneous in the context of the much longer afterglow. As pointed in the original fireball shock paper [98], the external shock bolometric luminosity builds up and decays as

$L \propto \begin{cases}t^{2} & \text { rise } \\ t^{-(1+q)} & \text { decay } .\end{cases}$

The first line is obtained equating, in the contact discontinuity frame, the kinetic flux $L / 4 \pi r^{2}$ to the external ram pressure $\rho_{\text {ext }} \Gamma^{2}$ during the initial phase where $\Gamma \sim$ constant, $r \propto t$, while the second follows from energy conservation $L \propto E / t$ under adiabatic conditions ( $q$ takes into account radiative effects or bolometric corrections; the flux per unit frequency rises in the same way, and decays with $q \geq 1$ in equ. (6)). At the deceleration radius (6) the fireball energy and the bulk Lorentz factor decrease by a factor $\sim 2$ over a timescale $t_{d e c} \sim r_{d e c} /\left(c \Gamma^{2}\right)$, and thereafter the bulk Lorentz factor decreases as a power law in radius. This is

$\Gamma \propto r^{-g} \propto t^{-g /(1+2 g)}, r \propto t^{1 /(1+2 g)}$,

with $g=(3,3 / 2)$ for the radiative (adiabatic) regime, in which $\rho r^{3} \Gamma \sim$ constant $\left(\rho r^{3} \Gamma^{2} \sim\right.$ constant). At late times, a similarity solution [6] solution with $g=7 / 2$ may be reached.

The spectrum of radiation is likely to be due to synchrotron radiation, whose peak frequency in the observer frame is $\nu_{m} \propto \Gamma B^{\prime} \gamma^{2}$, and both the comoving field $B^{\prime}$ and electron Lorentz factor $\gamma$ are likely to be proportional to $\Gamma$ [57]. This implies that as $\Gamma$ decreases, so will $\nu_{m}$, and the radiation will move to longer wavelengths. This led 76, 42 to early discussions, based on the forward blast wave, of the possibility of detecting at late times a radio or optical afterglow of the GRB. A 
more detailed treatment of the fireball dynamics indicate that approximately equal amounts of energy are radiated by the forward blast wave, moving with $\sim \Gamma$ into the surrounding medium, and by a reverse shock propagating with $\Gamma_{r}-1 \sim 1$ back into the ejecta [57]. The electrons are therefore shocked to much higher energies in the forward shock than in the reverse shock, producing a two-step synchrotron spectrum which during the deceleration time $t_{d e c}$ peaks in the optical (reverse) and in the $\gamma / X$ (forward) [59,60. The predicted fluences in the optical for typical bursts at cosmological distances were $\sim 10^{-7.5} \mathrm{erg} \mathrm{cm}^{-2}$ $\mathrm{s}^{-1}$, or about a 9th magnitude prompt optical flash [61] of duration comparable to the $\gamma$-rays, in agreement with a recent prompt optical detection in GRB 990123 [2]. Detailed calculations and predictions of the time evolution of such a forward and reverse shock afterglow model ([61]) preceded the observations of the first afterglow GRB970228 (14, 127]), which was detected in $\gamma$ rays, X-rays and several optical bands, and was followed up for a number of months.

The simplest spherical afterglow model generally concentrates only on the properties of the forward blast wave radiation, for which the flux at a given frequency and the synchrotron peak frequency decay at a rate 61,67

$F_{\nu} \propto t^{[3-2 g(1-2 \beta)] /(1+2 g)} \quad, \quad \nu_{m} \propto t^{-4 g /(1+2 g)}$,

where $g$ is the exponent of $\Gamma$ (equ. [7]) and $\beta$ is the photon spectral energy slope. The decay rate of the forward shock $F_{\nu}$ in equ.(8) is typically slower than that of the reverse shock [61], and the reason why the "simplest" model was stripped down to its forward shock component only is that, for the first two years 1997-1998, afterglows were followed in more detail only after the several hours needed by Beppo-SAX to acquire accurate positions, by which time both reverse external shock and internal shock components are expected to have become unobservable. This simple standard model has been remarkably successful at explaining the gross features and light curves of GRB 970228, GRB 970508 (after 2 days; for early rise, see $\S$ (5) e.g. 136, 121, 132,101. (see Figure 2).

This simplest afterglow model produces at any given time a three-segment power law spectrum

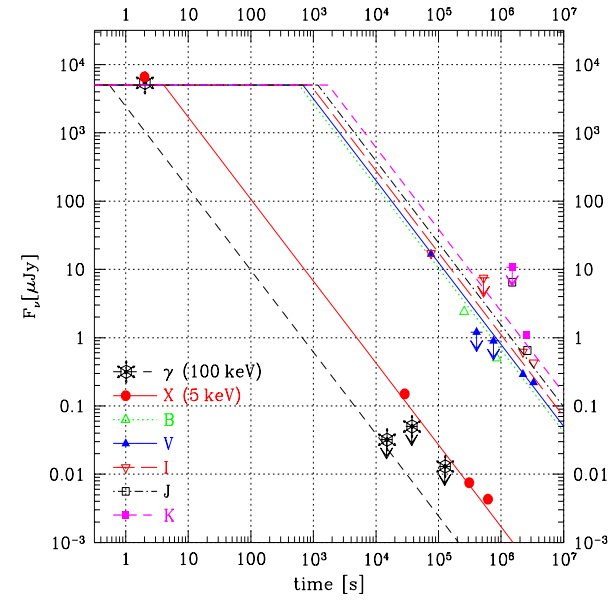

Figure 2. GRB 970228 light-curves compared [136] to the blast wave model predictions of 61

with two breaks. At low frequencies there is a steeply rising synchrotron self-absorbed spectrum up to a self-absorption break $\nu_{a}$, followed by a $+1 / 3$ energy index spectrum up to the synchrotron break $\nu_{m}$ corresponding to the minimum energy $\gamma_{m}$ of the power-law accelerated electrons, and then a $-(p-1) / 2$ energy spectrum above this break, for electrons in the adiabatic regime (where $\gamma^{-p}$ is the electron energy distribution above $\gamma_{m}$ ). A fourth segment and a third break is expected at energies where the electron cooling time becomes short compared to the expansion time, with a spectral slope $-p / 2$ above that. With this third "cooling" break $\nu_{b}$, first calculated in 65] and more explicitly detailed in [112], one has what has come to be called the simple "standard" model of GRB afterglows. One of the predictions of this model [61] is that the relation between the temporal decay index $\alpha$, for $g=3 / 2$ in $\Gamma \propto r^{-g}$, is related to the photon spectral energy index $\beta$ through through

$F_{\nu} \propto t^{\alpha} \nu^{\beta}$, with $\alpha=(3 / 2) \beta$.

This relationship appears to be valid in many (although not all) cases, especially after the first few days, and is compatible with an electron spectral index $p \sim 2.2-2.5$ which is typical of shock acceleration, e.g. 132,112,135, etc. As the remnant expands the photon spectrum moves to lower fre- 
quencies, and the flux in a given band decays as a power law in time, whose index can change as breaks move through it. For the simple standard model, snapshot overall spectra have been deduced by extrapolating spectra at different wavebands and times using assumed simple time dependences 133,135. These can be used to derive rough fits for the different physical parameters of the burst and environment, e.g. the total energy $E$, the magnetic and electron-proton coupling parameters $\epsilon_{B}$ and $\epsilon_{e}$ and the external density $n_{o}$ (see Figure 3).

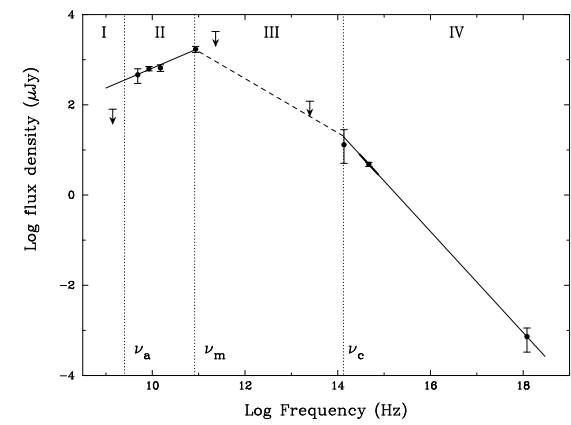

Figure 3. Snapshot spectrum of GRB 970508 at $t=12$ days and standard afterglow model fit 135

Since the simple afterglow model has generally proved quite robust, it is worth reviewing the assumptions made in it. The following apply both to the "simple standard" model using forward shocks only, and to the original 61 version including both forward and reverse shocks:

a) A single value of $E_{o}$ and $\Gamma_{o}=\eta$ is used,

b) the external medium $n_{\text {ext }}$ is homogeneous,

c) the accelerated electron spectral index $p$, the magnetic field and electron to proton equipartion ratios $\varepsilon_{B}$ and $\varepsilon_{e}$ do not change in time,

d) the expansion is relativistic and the dynamics are given by $\Gamma \propto r^{-3 / 2}$ (adiabatic),

d) the outflow is spherical (or angle independent inside some jet solid angle $\Omega_{j}$ ),

e) the observed radiation is characterized by the scaling relations along the line of sight.
These assumptions, even if correct over some range, clearly would break down after some time. Estimates for the time needed to reach the non-relativistic expansion regime are typically $\lesssim \operatorname{month}(\mathrm{s})(129)$, or less if there is an initial radiative regime $\Gamma \propto r^{-3}$. However, even when electron radiative times are shorter than the expansion time, it is unclear whether a regime $\Gamma \propto r^{-3}$ should occur, since it would require strong electron-proton coupling 65]. As far as sphericity, the standard model can be straightforwardly generalized to the case where the energy is assumed to be channeled initially into a solid angle $\Omega_{j}<4 \pi$ [58]. In this case 104 105] a change occurs after $\Gamma$ drops below $\Omega_{j}^{-1 / 2}$, after which the side of the jet becomes observable, and soon thereafter one expects a faster decay of $\Gamma$ if the jet starts to expands sideways, leading to a decrease in the brightness. A calculation based on the sideways expansion, using the usual scaling laws for a single central line of sight 105] leads then to a steepening of the light curve. Until recently, no evidence for a steepening could be found in afterglows over several months. E.g., in GRB 971214 [97], a snapshot standard model fit and the lack of a break in the late light curve could be, in principle, interpreted as evidence for lack of a jet, leading to an (isotropic) energy estimate of $10^{53.5}$ ergs. While such large energy outputs are possible in either NS-NS, NS-BH mergers [62] or in hypernova/collapsar models 81. 95] using MHD extraction of the spin energy of a disrupted torus and/or a central fast spinning $\mathrm{BH}$, it is worth stressing that what these snapshot fits constrain is only the energy per solid angle [66]. Also, the expectation of a break after some weeks or months (e.g., due to $\Gamma$ dropping either below a few, or below $\Omega_{j}^{-1 / 2}$ ) is based upon the simple impulsive (angle-independent delta or top-hat function) energy input approximation. The latter is useful, but departures from it would be natural, and certainly not surprising. In fact, as discussed below, tentative evidence for beaming in one obejct has recently been reported [51,27,.12], but it is difficulty to ascertain, and could be masked by a number of commonly expected effects. 


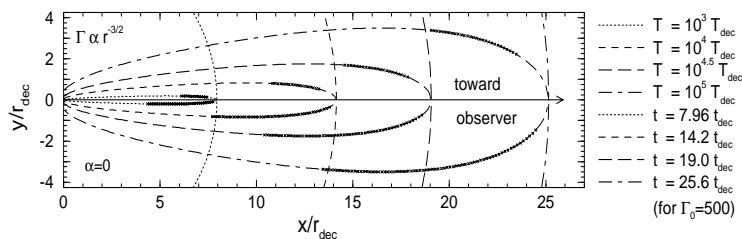

Figure 4. Ring-like equal-arrival time $T$ surfaces of an afterglow, based on 85.

\section{5. "Post-standard" Afterglow Models}

In a realistic situation, one could expect any of several fairly natural departures from the simple standard model to occur. The first one is that the emitting region seen by the observer resembles a ring [133, 84, 110 (see Figure 4). This effect may, in fact, be important in giving rise to the radio scintillation pattern seen in several afterglows, since this requires the emitting source to be of small dimensions, which is aided if the emission is ring-like, e.g. in the example of GRB 970508 [134 (Figure 5).

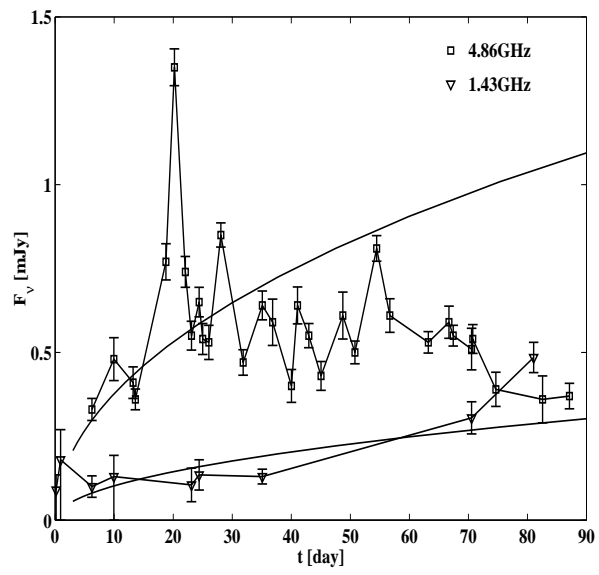

Figure 5. Radio afterglow light-curves of GRB970508 at $4.86 \mathrm{GHz}$ and $1.43 \mathrm{GHz}$, compared with the predictions of [134].

One expects afterglows to show a diversity in their decay rates, not only due to different $\beta$ but also from the possibility of a non-standard relation between the temporal decay index $\alpha$ and the spectral energy index $\beta$, different from equ. (9). The most obvious departure from the simplest standard model occurs if the external medium is inhomogeneous: for instance, for $n_{e x t} \propto r^{-d}$, the energy conservation condition is $\Gamma^{2} r^{3-d} \sim$ constant, which changes significantly the temporal decay rates 65. Such a power law dependence is expected if the external medium is a wind, say from an evolved progenitor star as implied in the hypernova scenario (such winds are generally used to fit supernova remnant models). Another obvious non-standard effect, which it is reasonable to expect, is departures from a simple impulsive injection approximation (i.e. from a delta or top hat function with a single value for $E_{o}$ and $\Gamma_{o}$ ). An example is if the mass and energy injected during the burst duration $t_{w}$ (say tens of seconds) obeys $M(>\Gamma) \propto \Gamma^{-s}, E(>\Gamma) \propto \Gamma^{1-s}$, i.e. more energy emitted with lower Lorentz factors at later times (but still shorter than the gamma-ray pulse duration). This would drastically change the temporal decay rate and extend the afterglow lifetime in the relativistic regime, providing a late "energy refreshment" to the blast wave on time scales comparable to the afterglow time scale [100]. These two cases lead to a decay rate

$\Gamma \propto r^{-g} \propto \begin{cases}r^{-(3-d) / 2} & ; n_{\text {ext }} \propto r^{-d} ; \\ r^{-3 /(2+s)} & ; E(>\Gamma) \propto \Gamma^{1-s} .\end{cases}$

Expressions for the temporal decay index $\alpha(\beta, s, d)$ in $F_{\nu} \propto t^{\alpha}$ are given by 65, 100], which now depend also on $s$ and/or $d$ (and not just on $\beta$ as in the simple standard relation of equ.(9). The result is that the decay can be flatter (or steeper, depending on $s$ and $d$ ) than the simple standard $\alpha=(3 / 2) \beta$. A third non-standard effect, which is entirely natural, occurs when the energy and/or the bulk Lorentz factor injected are some function of the angle. A simple case is $E_{o} \propto \theta^{-j}, \Gamma_{o} \propto \theta^{-k}$ within a range of angles; this leads to the outflow at different angles shocking at different radii and its radiation arriving at the observed at different delayed times, and it has a marked effect on the time dependence of the afterglow 65, with $\alpha=\alpha(\beta, j, k)$ flatter or steeper than the standard value, depending on $j, k$. Thus in general, a 


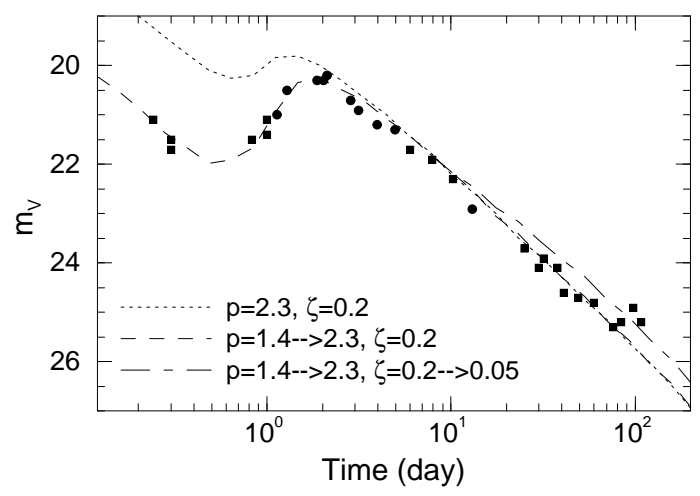

Figure 6. Optical light-curve of GRB 970508, fitted with a non-uniform injection model (a similar fit can be obtained with an off-axis jet plus a weaker isotropic component) 88

temporal decay index which is a function of more than one parameter

$F_{\nu} \propto t^{\alpha} \nu^{\beta}$, with $\alpha=\alpha(\beta, d, s, j, k, \cdots)$,

is not surprising; what is more remarkable is that, in many cases, the simple standard relation (9) is sufficient to describe the gross overall behavior at late times.

Strong evidence for departures from the simple standard model is provided by, e.g., sharp rises or humps in the light curves followed by a renewed decay, as in GRB 970508 ([89,93]). Detailed time-dependent model fits 88 to the Xray, optical and radio light curves of GRB 970228 and GRB 970508 show that, in order to explain the humps, a non-uniform injection (Figure 6) or an anisotropic outflow is required.

These fits indicate that the shock physics may be a function of the shock strength (e.g. the electron index $p$, injection fraction $\zeta$ and/or $\epsilon_{b}, \epsilon_{e}$ change in time), and also indicate that dust absorption is needed to simultaneously fit the X-ray and optical fluxes. The effects of beaming (outflow within a limited range of solid angles) can be significant [86], but are coupled with other effects, and a careful analysis is needed to disentangle them.

One consequence of "post-standard" decay laws (e.g. from density inhomogeneities, nonuniform injection or anisotropic outflow) is that the transition to a steeper jet regime $\Gamma<\theta_{j}^{-1} \sim$ few can occur as late as six months to a year after the outburst, depending on details of the energy input. This transition is made more difficult to detect by the fact that, as numerical integration over angles of the ring-like emission 85] show, the transition is very gradual and the effects of sideways expansion effects are not so drastic as inferred [105] from the scaling laws along the central line of sight. This is because even though the flux from the head-on part of the remnant decreases faster, this is more than compensated by the increased emission measure from sweeping up external matter over a larger angle, and by the fact that the extra radiation, arising at larger angles, arrives later and re-fills the steeper light curve. The inference (e.g. [97,105) that GRB 970508 and a few other bursts were isotropic due to the lack of an observable break is predicated entirely on the validity of the simplest standard fireball assumption. Since these assumptions are drastic simplifications, and physically plausible generalizations lead to different conclusions, one can interpret the results of 97,105 as arguments indicating that post-standard features are, in fact, necessary in some objects.

\section{Prompt multi-wavelength flashes, re- verse shocks and jets}

Prompt optical, X-ray and $\mathrm{GeV}$ flashes from reverse and forward shocks, as well as from internal shocks, have been calculated in theoretical fireball shock models for a number of years [59, 60, 82, 61, 113], as have been jets (e.g. 55,58, [60], and in more detail [104, 88, 86, 105]). Thus, while in recent years they were not explicitly part of the "simple standard" model, they are not strictly "post"-standard either, since they generally use the "standard" assumptions, and they have a long history. However, observational evidence for these effects were largely lacking, until the detection of a prompt (within $22 \mathrm{~s}$ ) optical flash from GRB 990123 with ROTSE by [2], together with X-ray, optical and radio follow-ups citekul99,gal99,fru99,and99,cas99,hjo99. GRB 
990123 is so far unique not only for its prompt optical detection, but also by the fact that if it were emitting isotropically, based on its redshift $z=1.6$ [51,1] its energy would be the largest of any GRB so far, $4 \times 10^{54}$ ergs. It is, however, also the first (tentative) case in which there is evidence for jet-like emission [51,27, 12]. An additional, uncommon feature is that a radio afterglow appeared after only one day, only to disappear the next 30,51.

The prompt optical light curve of GRB 990123 decays initially as $\propto t^{-2.5}$ to $\propto t^{-1.6}$ [2], much steeper than the typical $\propto t^{-1.1}$ of previous optical afterglows detected after several hours. However, after about 10 minutes its decay rate moderates, and appears to join smoothly onto a slower decay rate $\propto t^{-1.1}$ measured with large telescopes 30, 51,27,12 after hours and days. The prompt optical flash peaked at 9-th magnitude after $55 \mathrm{~s}$ [2], and in fact a 9-th magnitude prompt flash with a steeper decay rate had been predicted more than two years ago 61], from the synchrotron radiation of the reverse shock in GRB afterglows at cosmological redshifts (see also [113]). An optical flash contemporaneous with the $\gamma$-ray burst, coming from the reverse shock and with fluence corresponding to that magnitude, had also been predicted earlier [59,60]. An origin of the optical prompt flash in internal shocks [61,67] cannot be ruled out yet, but is less likely since the optical light curve and the $\gamma$-rays appear not to correlate well 114,30 (but the early optical light curve has only three points). The subsequent slower decay agrees with the predictions for the forward component of the external shock 61, 114, 67.

The evidence for a jet is possibly the most exciting, although must still be considered tentative. It is based on an apparent steepening of the light curve after about three days [51,27, 12]. This is harder to establish than the decay of the two previous earlier portions of the light curve, since by this time the flux has decreased to a level where the detector noise and the light of the host galaxy become important. However, after correcting for this, the r-band data appears to steepen significantly. (In the K-band, where the noise level is higher, a steepening is not obvi- ous, but the issue should be settled with further Space Telescope observations). If real, this steepening is probably due to the transition between early relativistic expansion, when the light-cone is narrower than the jet opening, and the late expansion, when the light-cone has become wider than the jet, leading to a drop in the effective flux 104, 51, 67, 105]. A rough estimate leads to a jet opening angle of 3-5 degrees, which would reduce the total energy requirements to about $4 \times 10^{52}$ ergs. This is about two order of magnitude less than the binding energy of a few solar rest masses, which, even allowing for substantial inefficiencies, is compatible with currently favored scenarios (e.g. 95.53]) based on a stellar collapse or a compact binary merger.

\section{Location and Environmental Effects}

The location of the afterglow relative to the host galaxy center can provide clues both for the nature of the progenitor and for the external density encountered by the fireball. A hypernova model would be expected to occur inside a galaxy in a high density environment $n_{o}>10^{3}-10^{5}$ $\mathrm{cm}^{-3}$. Most of the detected and well identified afterglows are inside the projected image of the host galaxy [7], and some also show evidence for a dense medium at least in front of the afterglow ([75]). For a number of bursts there are constraints from the lack of a detectable, even faint, host galaxy [116], but at least for BeppoSAX bursts (which is sensitive only to long bursts $\left.t_{b} \gtrsim 20 \mathrm{~s}\right)$ the success rate in finding candidate hosts is high.

In NS-NS mergers one would expect a BH plus debris torus system and roughly the same total energy as in a hypernova model, but the mean distance traveled from birth is of order several Kpc [10], leading to a burst presumably in a less dense environment. The fits of 135] to the observational data on GRB 970508 and GRB 971214 in fact suggest external densities in the range of $n_{o}=0.04-0.4 \mathrm{~cm}^{-3}$, which would be more typical of a tenuous interstellar medium. These could be within the volume of the galaxy, but for NS-NS on average one would expect as many GRB inside as outside. This is based on an estimate of the 
mean NS-NS merger time of $10^{8}$ years; other estimated merger times (e.g. $10^{7}$ years, 126]) would give a burst much closer to the birth site. BHNS mergers would also occur in timescales $\lesssim 10^{7}$ years, and would be expected to give bursts well inside the host galaxy ([10]; see however [28]). In at least one "snapshot" standard afterglow spectral fit for GRB 980329 [103] the deduced external density is $n_{o} \sim 10^{3} \mathrm{~cm}^{-3}$. In some of the other detected afterglows there is other evidence for a relatively dense gaseous environments, as suggested, e.g. by evidence for dust [102] in GRB970508, the absence of an optical afterglow and presence of strong soft X-ray absorption [33,69] in GRB 970828, the lack an an optical afterglow in the (radio-detected) afterglow ([122]) of GRB980329, and spectral fits to the low energy portion of the X-ray afterglow of several bursts [75]. The latter observations may be suggestive of hypernova models 81,28, involving the collapse of a massive star or its merger with a compact companion.

One important caveat is that all afterglows found so far are based on Beppo-SAX positions, which is sensitive only to long bursts $t_{b} \gtrsim 20 \mathrm{~s}$ [39]. This is significant, since it appears likely that NSNS mergers lead [53] to short bursts with $t_{b} \lesssim 10$ s. To make sure that a population of short GRB afterglows is not being missed will probably need to await results from HETE [35] and from the planned Swift [120] mission, which is designed to accurately locate $300 \mathrm{GRB} / \mathrm{yr}$.

An interesting case is the apparent coincidence of GRB 980425 with the unusual SN Ib/Ic 1998bw [29], which may represent a new class of SN [40,8]. If true, this could imply that some or perhaps all GRB could be associated with SN Ib/Ic [131], differring only in their viewing angles relative to a very narrow jet. Alternatively, the GRB could be (e.g. [138]) a new subclass of GRB with lower energy $E_{\gamma} \sim 10^{48}\left(\Omega_{j} / 4 \pi\right)$ erg, only rarely observable, while the great majority of the observed GRB would have the energies $E_{\gamma} \sim 10^{54}\left(\Omega_{j} / 4 \pi\right)$ ergs as inferred from high redshift observations. The difficulties are that it would require extreme collimations by factors $10^{-3}-10^{-4}$, and the statistical association is so far not significant 444].

The environment in which a GRB occurs should also influence the nature of the afterglows in other ways. The blast wave and reverse shock that give rise to the X-rays, optical, etc occur over timescales proportional to $t_{d e c} \propto n_{\text {ext }}^{-1 / 3}$ (equ. [6]) which is longer in lower density environments, so for the same energy the flux is lower, roughly $F_{\nu} \propto E_{o} n_{e x t}^{1 / 2}$, contributing also to make afterglows in the intergalactic medium harder to detect. However, in addition to affecting broadband fluxes, one may also expect specific spectral signatures from the external medium imprinted in the X-ray and optical continuum, such as atomic edges and lines [ [4, 91, 64]. These may be used both to diagnose the chemical abundances and the ionization state (or local separation from the burst), as well as serving as potential alternative redshift indicators. (In addition, the outflowing ejecta itself may also contribute blue-shifted edge and line features, especially if metal-rich blobs or filaments are entrained in the flow from the disrupted progenitor debris [63], which could serve as diagnostic for the progenitor composition and outflow Lorentz factor). To distinguish between progenitors (\$2), an interesting prediction (64]; see also [31, 11]) is that the presence of a measurable $\mathrm{Fe} \mathrm{K}-\alpha \mathrm{X}$-ray emission line could be a diagnostic of a hypernova, since in this case one may expect a massive envelope at a radius comparable to a light-day where $\tau_{T} \lesssim 1$, capable of reprocessing the X-ray continuum by recombination and fluorescence. Two groups 94, 139 have in fact recently reported the possible detection of Fe emission lines in GRB 970508 and GRB 970828.

\section{Conclusions}

The fireball shock model of gamma-ray bursts has proved quite robust in providing a consistent overall interpretation of the major features of these objects at various frequencies and over timescales ranging from the short initial burst to afterglows extending over many months. The standard internal shock scenario is able to reproduce the properties of the $\gamma$-ray light curves, while external shocks involving a forward blast wave and a reverse shock are successful in reproducing the afterglows observed in X-rays, optical and radio. The "simple standard model" of afterglows, involving four spectral slopes and 
three breaks is quite useful in understanding the 'snapshot' multiwavelength spectra of most afterglows. However, the effects associated with a jetlike outflow and the possible differential beaming at various energies requires further investigations, both theoretical and observational. Caution is required in interpreting the observations on the basis of the simple standard model. For instance, more detailed numerical models, as opposed to the more common analytical scaling law models, show that the contributions of radiation from different angles and the gradual transition between different dynamical and radiative regimes lead to a considerable rounding-off of the spectral shoulders and light-curve slope changes, so that breaks cannot be easily located unless the spectral sampling is dense and continuous, both in frequency and in time. Some of the observed light curves with humps, e.g. in GRB 970508, require 'post-standard' model features (i.e. beyond those assumed in the standard model), such as either non-uniform injection episodes or anisotropic outflows. Time-dependent multiwavelength fits 88 of some bursts also indicate that the parameters characterizing the shock physics change with time. Even without humps or slope changes, a non-standard relation between the spectral and temporal decay slope is observed in several objects, e.g. in GRB 990123 [51]. These are, in our view [67], a strong indication for "post-standard" effects in such bursts.

Much progress has been made in understanding how gamma-rays can arise in fireballs produced by brief events depositing a large amount of energy in a small volume, and in deriving the generic properties of the long wavelength afterglows that follow from this. There still remain a number of mysteries, especially concerning the identity of their progenitors, the nature of the triggering mechanism, the transport of the energy, the time scales involved, and the nature and effects of beaming. However, even if we do not yet understand the details of the gamma-ray burst central engine, it is clear that these phenomena are among the most powerful transients in the Universe, and they could serve as powerful beacons for probing the high redshift $(z>5)$ universe. The modeling of the burst mechanism itself, as well as the resulting outflows and radiation, will continue to be a formidable challenge to theorists and to computational techniques. However, the theoretical understanding appears to be converging, and with dedicated new and planned observational missions under way, the prospects for significant progress are realistic.

I am grateful to Martin Rees for stimulating collaborations, as well as to Alin Panaitescu, Hara Papathanassiou and Ralph Wijers.

\section{REFERENCES}

1. Andersen et al. 1999, Science, March 26 issue

2. Akerlof, C., et al., 1999, Nature, in press (astro-ph/9903271)

3. Band, D., et al., 1993, Ap.J., 413, 281

4. Bisnovatyi-Kogan, G \& Timokhin, A, 1997, Astr. Rep. 41, 423

5. Blandford, R.D. \& Znajek, R.L., 1977, MNRAS, 179, 433

6. Blandford, R.D. \& McKee, C., 1976, Phys.Fluids 19, 1130

7. Bloom, J., etal, 1998, A\& A Supp.,in press (Procs. Rome Conference on GRB)

8. Bloom, J, et al., 1998, ApJ 506, L105

9. Bloom, J., etal, 1998, ApJ 507, L25

10. Bloom, J., Sigurdsson, S. \& Pols, O., 1999, MNRAS in press (astro-ph/9805222)

11. Böttcher, $\mathrm{M}$, et al., 1998, astro-ph/9809156

12. Castro-Tirado et al., 1999, Science, March 26 issue

13. Connors, A \& Hueter, G.J. 1998, ApJ 501, 307

14. Costa, E., et al., 1997, Nature, 387, 783

15. Daigne, F \& Mochkovitch, R, 1998, MNRAS, 296, 275

16. Dermer, C \& Mitman, K, 1998, astro$\mathrm{ph} / 9809411$

17. Djorgovski, S.G. et al., 1997, IAUC 6660

18. Djorgovski, S.G. et al., 1998, ApJ 508, L17

19. Duncan, R \& Thompson, C, 1992, ApJ, 392, L9

20. Eichler, D., Livio, M., Piran, T. and Schramm, D.N., 1989, Nature, 340, 126

21. Fenimore, E, Madras, C, and Nayakshin, S, 1996, ApJ, 473, 998

22. Fenimore, E, et al., 1998, ApJ in press (astro- 
$\mathrm{ph} / 9802200)$

23. Fishman, G. \& Meegan, C., 1995, Ann.Rev Astr.Ap.,33, 415

24. Frail, D, et al., 1997, Nature, 389, 261

25. Frail, D, A\& A Supp.,in press (Procs. Rome Conference on GRB)

26. Fruchter, A, astro-ph/9810224

27. Fruchter, A. etal, Ap.J. submitted astro$\mathrm{ph} / 9902236$ )

28. Fryer, C \& Woosley, S, 1998, ApJ(Lett) subm astro-ph/9804167

29. Galama, T. et al., 1998, Nature, in press (astro-ph/9806175)

30. Galama, T. et al., 1999, Nature, in press (astro-ph/9903021)

31. Ghisellini, G, et al., 198, astro-ph/9808156

32. Goodman, J., 1986, ApJ, 1986, 308, L47

33. Groot, P. et al., 1997; in Gamma-Ray Bursts, Meegan, C., Preece, R. \& Koshut, T., eds., 1997 (AIP: New York), p. 557

34. Harding, A.K. and Baring, M.G., 1994, in Gamma-ray Bursts, ed. G. Fishman, et al., p. 520 (AIP 307, NY)

35. HETE, http://space.mit.edu/HETE/

36. Hjorth, etal, 1999, Science, March 26 issue

37. Horack, J. M., Mallozzi, R. S., \& Koshut, T. M. 1996, ApJ, 466, 21

38. Hurley, K., et al., 1994, Nature, 372,652

39. Hurley, K., A\& A Supp.,in press (Procs. Rome Conference on GRB)

40. Iwamoto, K, et al., 1998, Nature 395, 672

41. Katz, J., 1994a, ApJ, 422, 248

42. Katz, J., 1994b, ApJ, 432, L107

43. Katz, J.I., 1997, ApJ, 490, 633

44. Kippen, R.M. et al., 1998, ApJ subm (astro$\mathrm{ph} / 9806364)$

45. Klebesadel, R, Strong, I \& Olson, R, 1973, ApJ, 182, L85

46. Kluzniak, W \& Ruderman, M, 1998, ApJ 508, L113

47. Kobayashi, S, Piran, T \& Sari, R, 1998, ApJ, 490, 92

48. Kouveliotou, C., et al., 1993, Ap.J., 413, L101

49. Kouveliotou, C., 1998, review at the APS Spring mtg, Indianapolis, IN

50. Kulkarni, S., et al., 1998, Nature, 393, 35

51. Kulkarni, S., et al., 1999, Nature, in press (astro-ph/9902272)
52. Mazets, EP, Golenetskii, SV \& Ilinskii, VN, 1974, JETP Lett. 19, 77

53. Macfadyen, A \& Woosley, S, 1999, ApJ n press (astro-ph/9810274)

54. Mészáros , P., 1995, 17th Texas Symp. Relativistic Astrophysics, H. Böhringer et al, N.Y. Acad. Sci., 440

55. Mészáros , P \& Rees, M.J., 1992, ApJ, 397, 570

56. Mészáros , P \& Rees, MJ, 1992b, MNRAS, 257, 29P

57. Mészáros, P. \& Rees, M.J., 1993a, ApJ, 405, 278

58. Mészáros , P., Laguna, P \& Rees, M.J., 1993, ApJ, 415, 181

59. Mészáros , P. and Rees, M.J., 1993b, Ap.J., 418, L59

60. Mészáros , P., Rees, M.J. \& Papathanassiou, H, 1994, Ap.J., 432, 181

61. Mészáros , P \& Rees, M.J., 1997a, ApJ, 476, 232

62. Mészáros , P \& Rees, M.J., 1997b, ApJ, 482, L29

63. Mészáros, P \& Rees, M.J., 1998a, ApJ, 502, L105

64. Mészáros , P \& Rees, M.J., 1998b, MNRAS, 299, L10

65. Mészáros , P, Rees, M.J. \& Wijers, R, 1998, Ap.J., 499, 301 (astro-ph/9709273)

66. Mészáros , P, Rees, M.J. \& Wijers, R, 1998b, New Astron, in press (astro-ph/9808106)

67. Mészáros, P \& Rees, M.J., MNRAS, in press (astro-ph/9902367)

68. Metzger, M et al., 1997, Nature, 387, 878

69. Murakami, T. et al., 1997, in Gamma-Ray Bursts, Meegan, C., Preece, R. \& Koshut, T., eds., 1997 (AIP: New York), p. 435

70. Narayan, R., Piran, T. \& Shemi, A, 1991, ApJ, 379, L17

71. Narayan, R., Paczyński , B. \& Piran, T., 1992, Ap.J., 395, L83

72. Norris, J, 1998, private communication

73. Norris, J. P., et al. 1995, ApJ, 439, 542

74. Odewahn, S, et al., 1998, ApJ, 509, L50

75. Owen, A., et al, 1998, Astron.\&Astrophys. in press (astro-ph/9809356),

76. Paczyński, B. \& Rhoads, J, 1993, Ap.J., 418, L5 
77. Paczyński , B. \& Xu, G., 1994, ApJ, 427, 708

78. Paczyński , B., 1986, ApJ, 308, L43

79. Paczyński , B., 1990, Ap.J., 363, 218

80. Pacziński, B., 1991, Acta. Astron., 41, 257

81. Paczyński , B., 1998, ApJ, 494, L45

82. Papapathanassiou, H \& Mészáros , P, 1996, ApJ, 471, L91

83. Panaitescu, A \& Mészáros , P, 1998a, ApJ, 492, 683

84. Panaitescu, A. \& Mészáros, P., 1998b, ApJ, 501, 772

85. Panaitescu, A. \& Mészáros , P., 1998, ApJ, 493, L31

86. Panaitescu, A. \& Mészáros , P., 1999, ApJ, subm. (astro-ph/9806016)

87. Panaitescu, A. \& Mészáros, P., 1998d, ApJ, subm (astro-ph/9810258)

88. Panaitescu, A, Mészáros , P \& Rees, MJ, 1998, ApJ, 503, 314

89. ApJ 496 (1998) 311

90. Pendleton, G, et al., 1996, Ap[J, 464, 606

91. Perna, R. \& Loeb, A., 1998, ApJ, 503, L135

92. Phinney, E.S., 1991, ApJ, 380, L17

93. Piro, L, et al., 1998, A \& A, 331, L41

94. Piro, L, et al., 1998b, A\& A Supp.,in press (Procs. Rome Conference on GRB)

95. Popham, R., Woosley, S \& Fryer, C., 1998, ApJ subm (astro-ph/9807028)

96. Pugliese, G, Falk, H \& Biermann, P, 1999, A\&A, in press (astro-ph/9903036)

97. Ramprakash, A et al., 1998, Nature 393, 38

98. Rees, M.J. \& Mészáros, P., 1992, MNRAS, 258, P41

99. Rees, M.J. \& Mészáros, P., 1994, ApJ, 430, L93

100Rees, M.J. \& Mészáros , P., 1998, ApJ, 496, L1

101Reichart, D., 1997, ApJ, 485, L57

102Reichart, D., 1998, ApJ, 495, L99

103Reichart, D \& Lamb, D.Q., 1998, A\& A Supp,in press (Procs. Rome Conf. on GRB)

104Rhoads, J, 1997, Ap.J., 487, L1

105Rhoads, J, 1999, ApJ subm, astro$\mathrm{ph} / 9903383$

106Ruffert, M., Janka, H.-T., Takahashi, K., Schaefer, G., 1997, A\& A, 319, 122

107Ruffert, M. \& Janka, H.-T., 1998, A\&A subm (astro-ph/9804132)
108.Sahu, K., et al., 1997, Nature 387, 476

109.Sari, R. \& Piran, T., 1995, ApJ, 455, L143

110.Sari, R, 1998, ApJ, 494, L49

111.Sari, R \& Piran, T, 1998, ApJ, 485, 270

112.Sari, R , Piran, T \& Narayan, R, 1998, ApJ, 497, L17 (astro-ph/9712005)

113.Sari, R \& Piran, T., 1999a, A\&A submitted (astro-ph/9901105

114.Sari, R \& Piran, T, 1999b, Ap.J. submitted (astro-ph/9902009)

115.Schaefer, B.E. et al, 1997, ApJ, 489, 693

116.Schaefer, B.E., 1998, ApJ in press astro $\mathrm{ph} / 9810424$ )

117.Shemi, A. and Piran, T., 1990, ApJ, 365, L55

118.Spruit, H, 1999, A\&A, in press (astro$\mathrm{ph} / 98 \ldots)$

119.Strohmeyer, T, et al., 1998, ApJ, 500, 873

120.Swift, http://swift.gsfc.nasa.gov

121.Tavani, M., 1997, ApJ, 483, L87

122.Taylor, G.B., et al., 1997, Nature, 389, 263

123.Thompson, C., 1994, MNRAS, 270, 480

124.Totani, T, 1997, ApJ, 486, L71

125.Usov, V.V., 1994, MNRAS, 267, 1035

126.van den Heuvel, E, in X-ray Binaries \& Recycled Pulsars (ed. E.van den Heuvel and S.Rappaport), Kluwer, 1992, pp 233

127.van Paradijs, J, et al., 1997, Nature,386, 686

128.van Paradijs, J, 1998, invited review at APS Spring mtg, Indianapolis, IN

129.Vietri, M., 1997a, ApJ, 478, L9

130.Vietri, M. \& Stella, L, 1998, ApJ, in press

131.Wang, L. \& Wheeler, J.C., 1998, ApJ subm (astro-ph/9806212

132.Waxman, E., 1997, ApJ, 485, L5

133.Waxman, E., 1997b, ApJ, 489, L33

134.Waxman, E., Kulkarni, S \& Frail, D, 1998, ApJ, 497, 288

135.Wijers, R.A.M.J. \& Galama, T., 1998, ApJ, subm (astro-ph/9805341)

136.Wijers, R.A.M.J., Rees, M.J. \& Mészáros , P., 1997, MNRAS, 288, L51

137.Woosley, S., 1993, Ap.J., 405, 273

138.Woosley, S., Eastman, R. \& Schmidt, B., 1998, ApJ, subm (astro-ph/9806299)

139.Yoshida, A, et al., 1998, A\& A Supp.,in press (Procs. Rome Conference on GRB) 